Kragujevac Journal of Mathematics

Volume 45(6) (2021), Pages 943-950.

\title{
SOME RESULTS FOR ENDOMORPHISMS IN PRIME RINGS
}

\author{
ABDELKARIM BOUA ${ }^{1}$
}

\begin{abstract}
In this article, we present some commutativity theorems for a prime ring $\mathcal{R}$ equipped with endomorphisms $\alpha, \beta, \gamma$ and $\delta$ satisfying any one of the following identities:

(1) $[\alpha(x), \beta(y)]+\gamma([x, y])+\delta(x \circ y)=0$ for all $x, y \in \mathcal{R}$;

(2) $\alpha(x) \circ \beta(y)+\gamma([x, y])=0$ for all $x, y \in \mathcal{R}$.

Moreover, we provide examples to show that the assumed restrictions cannot be relaxed.
\end{abstract}

\section{INTRODUCTION}

Let $\mathcal{R}$ be a ring with center $Z(\mathcal{R})$. For any $x, y \in \mathcal{R},[x, y]$ will denote the commutator $x y-y x$ while $x \circ y$ will represent the anti-commutator $x y+y x$. Recall that a ring $\mathcal{R}$ is said to be prime if $a \mathcal{R} b=\{0\}$ implies that either $a=0$ or $b=0$. A ring $\mathcal{R}$ is said to be 2 -torsion free if $2 a=0$ (where $a \in \mathcal{R}$ ) implies $a=0$. It is straight forward to see that a prime ring with characteristic different from two is 2-torsion free. A mapping $f: \mathcal{R} \rightarrow \mathcal{R}$ is said to be centralizing on $\mathcal{R}$ if $[f(x), x] \in Z(\mathcal{R})$ holds for all $x \in \mathcal{R}$. In the special case if $[f(x), x]=0$ for all $x \in \mathcal{R}, f$ is said to be commuting on $\mathcal{R}$. An additive mapping $d: \mathcal{R} \rightarrow \mathcal{R}$ is said to be a derivation of $\mathcal{R}$ if $d(x y)=d(x) y+x d(y)$ for all $x, y \in \mathcal{R}$. A derivation $d$ is said to be inner if there exists $a \in \mathcal{R}$ such that $d(x)=a x-x a$ for all $x \in \mathcal{R}$. Following Bresar [6], an additive mapping $F: \mathcal{R} \rightarrow \mathcal{R}$ is called a generalized derivation if there exists a derivation $d: \mathcal{R} \rightarrow \mathcal{R}$ such that $F(x y)=F(x) y+x d(y)$ holds for all $x, y \in \mathcal{R}$. The concept of generalized derivations includes both the concept of derivation and the concept of left multiplier (i.e., an additive mapping $F: \mathcal{R} \rightarrow \mathcal{R}$ satisfying $F(x y)=F(x) y$ for all $x, y \in \mathcal{R})$.

Key words and phrases. Prime ring, endomorphisms, commutativity.

2010 Mathematics Subject Classification. Primary: 16N60, 15A27. Secondary: 16S50.

DOI 10.46793/KgJMat2106.943B

Received: April 07, 2019.

Accepted: July 08, 2019. 
Recently, a considerable number of researchers have investigated the ideals in prime rings as well as the commutativity of prime rings that consider derivations and generalized derivations, see for example [1-3] and [4].

Over the last four decade, several authors have proved results on commutativity of prime rings or semiprime rings that admitting automorphisms, derivations or generalized derivations which are centralizing or commuting on appropriate subset of $\mathcal{R}$ (see $[2-5]$ etc.).

In this paper, we investigate the commutativity of a prime ring $\mathcal{R}$ admitting endomorphisms $\alpha, \beta, \gamma$ and $\delta$ satisfying any one of the following properties:

(a) $[\alpha(x), \beta(y)]+\gamma([x, y])+\delta(x \circ y)=0$ for all $x, y \in \mathcal{R}$;

(b) $\alpha(x) \circ \beta(y)+\gamma([x, y])=0$ for all $x, y \in \mathcal{R}$.

\section{Some PRELIMINARIES}

This section, includes some well known basic identities which will be used for developing the proof of our main results:

(a) $[x, y z]=y[x, z]+[x, y] z$ for all $x, y, z \in \mathcal{R}$;

(b) $[x y, z]=x[y, z]+[x, z] y$ for all $x, y, z \in \mathcal{R}$;

(c) $x \circ(y z)=(x \circ y) z-y[x, z]=y(x \circ z)+[x, y] z$ for all $x, y, z \in \mathcal{R}$;

(d) $(x y) \circ z=x(y \circ z)-[x, z] y=(x \circ z) y+x[y, z]$ for all $x, y, z \in \mathcal{R}$.

\section{Some Results for Prime Rings}

Theorem 3.1. Let $\mathcal{R}$ be a prime ring with $\operatorname{char}(\mathcal{R}) \neq 2, \alpha, \beta, \gamma$ and $\delta$ endomorphisms of $\mathcal{R}$ such that

$$
[\alpha(x), \beta(y)]+\gamma([x, y])+\delta(x \circ y)=0, \quad \text { for all } x, y \in \mathcal{R} .
$$

If $\beta, \gamma$ are onto, then $\delta=0$ and $\mathcal{R}$ is commutative.

Proof. Suppose that

$$
[\alpha(x), \beta(y)]+\gamma([x, y])+\delta(x \circ y)=0, \quad \text { for all } x, y \in \mathcal{R} .
$$

Replacing $y$ by $y x$ in (3.1), we get

$\beta(y)[\alpha(x), \beta(x)]+[\alpha(x), \beta(y)] \beta(x)+\gamma([x, y]) \gamma(x)+\delta(x \circ y) \delta(x)=0, \quad$ for all $x, y \in \mathcal{R}$.

For $y=x,(3.1)$ implies that

$$
[\alpha(x), \beta(x)]+2 \delta\left(x^{2}\right)=0, \quad \text { for all } x \in \mathcal{R} .
$$

Using (3.1) and (3.3), then (3.2) can be rewritten as

(3.4) $2 \beta(y) \delta\left(x^{2}\right)=\gamma([x, y])(\gamma(x)-\beta(x))+\delta(x \circ y)(\delta(x)-\beta(x)), \quad$ for all $x, y \in \mathcal{R}$. 
For $y=x,(3.4)$ gives

$$
\beta(x) \delta\left(x^{2}\right)=\delta\left(x^{2}\right)(\delta(x)-\beta(x))=\delta\left(x^{2}\right) \delta(x)-\delta\left(x^{2}\right) \beta(x), \quad \text { for all } x \in \mathcal{R} .
$$

Taking $x y$ in place of $y$ in (3.4), it is obvious to see that

$$
\begin{aligned}
2 \beta(x) \beta(y) \delta\left(x^{2}\right)= & \gamma(x) \gamma([x, y])(\gamma(x)-\beta(x)) \\
& +\delta(x) \delta(x \circ y)(\delta(x)-\beta(x)), \quad \text { for all } x, y \in \mathcal{R} .
\end{aligned}
$$

Left-multiplying (3.4) by $\beta(x)$, we have also

$$
\begin{aligned}
2 \beta(x) \beta(y) \delta\left(x^{2}\right)= & \beta(x) \gamma([x, y])(\gamma(x)-\beta(x)) \\
& +\beta(x) \delta(x \circ y)(\delta(x)-\beta(x)), \quad \text { for all } x, y \in \mathcal{R} .
\end{aligned}
$$

By identifying (3.6) and (3.7), we can easily arrive at

$$
(\gamma(x)-\beta(x)) \gamma([x, y])(\gamma(x)-\beta(x))+(\delta(x)-\beta(x)) \delta(x \circ y)(\delta(x)-\beta(x))=0 .
$$

For $x=y$, using $\operatorname{char}(\mathcal{R}) \neq 2$, then

$$
(\delta(x)-\beta(x)) \delta\left(x^{2}\right)(\delta(x)-\beta(x))=0, \quad \text { for all } x \in \mathcal{R} .
$$

Using (3.5) and (3.8), we obtain

$$
(\delta(x)-\beta(x)) \beta(x) \delta\left(x^{2}\right)=0, \quad \text { for all } x \in \mathcal{R},
$$

and

$$
(\delta(x)-\beta(x)) \delta\left(x^{2}\right) \delta(x)=(\delta(x)-\beta(x)) \delta\left(x^{2}\right) \beta(x), \quad \text { for all } x \in \mathcal{R} .
$$

Right-multiplying (3.4) by $\beta(x) \delta\left(x^{2}\right)$ and using (3.9), we get

$$
2 \beta(y) \delta\left(x^{2}\right) \beta(x) \delta\left(x^{2}\right)=\gamma([x, y])(\gamma(x)-\beta(x)) \beta(x) \delta\left(x^{2}\right), \quad \text { for all } x, y \in \mathcal{R} .
$$

Replacing $y$ by $x y$ in (3.10), we can easily arrive at

$$
(\gamma(x)-\beta(x)) \gamma([x, y])(\gamma(x)-\beta(x)) \beta(x) \delta\left(x^{2}\right)=0, \quad \text { for all } x, y \in \mathcal{R} .
$$

Using (3.10) and (3.11), we find that

$$
(\gamma(x)-\beta(x)) \beta(y) \delta\left(x^{2}\right) \beta(x) \delta\left(x^{2}\right)=0, \quad \text { for all } x, y \in \mathcal{R} .
$$

Since $\beta$ is onto, we get

$$
(\gamma(x)-\beta(x)) \mathcal{R} \delta\left(x^{2}\right) \beta(x) \delta\left(x^{2}\right)=\{0\}, \quad \text { for all } x \in \mathcal{R} .
$$

By primeness of $\mathcal{R}$, we obtain

$$
\gamma(x)=\beta(x) \text { or } \delta\left(x^{2}\right) \beta(x) \delta\left(x^{2}\right)=0 \quad \text { for all } x \in \mathcal{R} .
$$

Suppose there exists $x_{0} \in \mathcal{R}$ such that $\gamma\left(x_{0}\right)=\beta\left(x_{0}\right)$, then (3.4) becomes

$$
2 \beta(y) \delta\left(x_{0}^{2}\right)=\delta\left(x_{0} \circ y\right)\left(\delta\left(x_{0}\right)-\beta\left(x_{0}\right)\right), \quad \text { for all } y \in \mathcal{R} \text {. }
$$

In (3.13) we substitute $x_{0} y$ for $y$ and using $\operatorname{char}(\mathcal{R}) \neq 2$, to get

$$
\left(\delta\left(x_{0}\right)-\beta\left(x_{0}\right)\right) \beta(y) \delta\left(x_{0}^{2}\right)=0, \quad \text { for all } y \in \mathcal{R} .
$$

Since $\beta$ is onto, we obtain $\left(\delta\left(x_{0}\right)-\beta\left(x_{0}\right)\right) \mathcal{R} \delta\left(x_{0}^{2}\right)=\{0\}$. By primeness of $\mathcal{R}$, we conclude that either $\delta\left(x_{0}\right)=\beta\left(x_{0}\right)$ or $\delta\left(x_{0}^{2}\right)=0$. 
If $\delta\left(x_{0}\right)=\beta\left(x_{0}\right)$, according to our assumption after (3.12) it follows from (3.4) that $2 \beta(y) \delta\left(x_{0}^{2}\right)=0$ for all $y \in \mathcal{R}$. Since $\beta$ is onto and $\operatorname{char}(\mathcal{R}) \neq 2$, we conclude that $\delta\left(x_{0}^{2}\right)=0$. In both cases, we have $\delta\left(x_{0}^{2}\right)=0$ and by (3.12), we get

$$
\delta\left(x^{2}\right) \beta(x) \delta\left(x^{2}\right)=0, \quad \text { for all } x \in \mathcal{R} .
$$

Using (3.5), we conclude that

$$
0=\delta\left(x^{2}\right) \beta(x) \delta\left(x^{2}\right) \delta\left(x^{2}\right)=\delta\left(x^{2}\right)\left(\delta\left(x^{2}\right) \delta(x)-\delta\left(x^{2}\right) \beta(x)\right) \delta\left(x^{2}\right),
$$

which leads to $0=\delta\left(x^{2}\right) \delta\left(x^{2}\right) \delta(x) \delta\left(x^{2}\right)=\delta\left(x^{7}\right)=(\delta(x))^{7}$ for all $x \in \mathcal{R}$. By a wellknow result of Lovitzki [7] a prime rings cannot be nil of bounded index. Then $\delta=0$. In this case, equation (3.4) becomes

$$
\gamma([x, y])(\gamma(x)-\beta(x))=0, \quad \text { for all } x, y \in \mathcal{R} .
$$

Taking ty in place of $y$ in (3.14), and using it again, we obtain

$$
\gamma([x, y]) \gamma(t)(\gamma(x)-\beta(x))=0, \quad \text { for all } x, y, t \in \mathcal{R} .
$$

Since $\gamma$ is onto, we get $\gamma([x, y]) \mathcal{R}(\gamma(x)-\beta(x))=\{0\}$, for all $x, y \in \mathcal{R}$. In view of the primeness of $\mathcal{R}$, the last equation reduces to

$$
\gamma([x, y])=0 \quad \text { or } \quad \gamma(x)=\beta(x), \quad \text { for all } x, y \in \mathcal{R} .
$$

If there exists $x_{0} \in \mathcal{R}$ such that $\gamma\left(\left[x_{0}, y\right]\right)=0$ for all $y \in \mathcal{R}$, it is clear that $\gamma\left(x_{0}\right) \in Z(\mathcal{R})$ because $\gamma$ is onto and so, $\left[\alpha(x), \beta\left(x_{0}\right)\right]=0$ for all $x \in \mathcal{R}$.

By hypothesis, we have

$$
\left[\alpha(x), \beta\left(y x_{0}\right)\right]+\gamma\left(\left[x, y x_{0}\right]\right)=0, \quad \text { for all } x, y \in \mathcal{R},
$$

which leads to

(3.16) $\beta(y)\left[\alpha(x), \beta\left(x_{0}\right)\right]+[\alpha(x), \beta(y)] \beta\left(x_{0}\right)+\gamma([x, y]) \gamma\left(x_{0}\right)=0, \quad$ for all $x, y \in \mathcal{R}$.

Since $\left[\alpha(x), \beta\left(x_{0}\right)\right]=0$ for all $x \in \mathcal{R},(3.16)$ becomes

$$
[\alpha(x), \beta(y)] \beta\left(x_{0}\right)+\gamma([x, y]) \gamma\left(x_{0}\right)=0, \quad \text { for all } x, y \in \mathcal{R} .
$$

Using (3.1), the last equation yields

$$
\gamma([x, y])\left(\gamma\left(x_{0}\right)-\beta\left(x_{0}\right)\right)=0, \quad \text { for all } x, y \in \mathcal{R} .
$$

Replacing $y$ by $y t$ in (3.17) and using it with the fact that $\gamma$ is onto, we conclude that $\gamma([x, y]) \mathcal{R}\left(\gamma\left(x_{0}\right)-\beta\left(x_{0}\right)\right)=\{0\}$, for all $x, y \in \mathcal{R}$. Since $\mathcal{R}$ is prime, we obtain $\gamma([x, y])=0$ for all $x, y \in \mathcal{R}$ or $\gamma\left(x_{0}\right)=\beta\left(x_{0}\right)$. Therefore, $[x, y]=0$ for all $x, y \in \mathcal{R}$ or $\gamma\left(x_{0}\right)=\beta\left(x_{0}\right)$. In this case, (3.15) forces that $\mathcal{R}$ is commutative or $\gamma(x)=\beta(x)$ for all $x \in \mathcal{R}$.

Now assume that the second case, then (3.1) becomes

$$
[\alpha(x), \beta(y)]+\beta([x, y])=0, \quad \text { for all } x, y \in \mathcal{R} .
$$

Taking $x y$ instead of $x$ in (3.18), we obtain

$$
\beta([x, y])(\beta(y)-\alpha(y))=0, \quad \text { for all } x, y \in \mathcal{R} .
$$


Putting $x r$ in place of $x$ where $r \in \mathcal{R}$, we can easily arrive at

$$
\beta([x, y]) \mathcal{R}(\beta(y)-\alpha(y))=\{0\}, \quad \text { for all } x, y \in \mathcal{R} .
$$

In light of primeness of $\mathcal{R}$, we arrive at

$$
\beta([x, y])=0 \text { or } \alpha(y)=\beta(y), \quad \text { for all } x, y \in \mathcal{R} .
$$

If there exists $y_{0} \in \mathcal{R}$ such that $\alpha\left(y_{0}\right)=\beta\left(y_{0}\right)$, by (3.18) we have

$$
\begin{aligned}
0 & \left.\left.=\left[\alpha\left(y_{0}\right), \beta(x)\right]\right)+\beta\left(\left[y_{0}, x\right]\right)=\left[\beta\left(y_{0}\right), \beta(x)\right]\right)+\beta\left(\left[y_{0}, x\right]\right) \\
& =2 \beta\left(\left[y_{0}, x\right]\right), \quad \text { for all } x \in \mathcal{R} .
\end{aligned}
$$

Since $\operatorname{char}(\mathcal{R}) \neq 2$, we get $\beta\left(\left[y_{0}, x\right]\right)=0$ for all $x \in \mathcal{R}$. Then (3.19) becomes $\beta([x, y])=0$ for all $x, y \in \mathcal{R}$. Since $\beta$ is onto, then $[x, y]=0$ for all $x, y \in \mathcal{R}$, which forces that $\mathcal{R}$ is commutative.

Corollary 3.1. Let $\mathcal{R}$ be a prime ring with $\operatorname{char}(\mathcal{R}) \neq 2$ and $\alpha$, $\beta$ endomorphisms of $\mathcal{R}$ such that $\beta$ is onto, then the following assertions are equivalent:

(a) $[\alpha(x), \beta(y)]+\beta([x, y])=0$ for all $x, y \in \mathcal{R}$;

(b) $\mathcal{R}$ is commutative.

Proof. Just replace $\gamma$ by $\beta$ and $\delta$ with the null application in Theorem 3.1.

Corollary 3.2. Let $\mathcal{R}$ be a prime ring with $\operatorname{char}(\mathcal{R}) \neq 2$ and $\alpha$ an endomorphism of $\mathcal{R}$, then the following assertions are equivalent:

(a) $\alpha(x)+x \in Z(\mathcal{R})$ for all $x \in \mathcal{R}$;

(b) $\mathcal{R}$ is commutative.

Proof. If we put $\beta=i d_{\mathcal{R}}$, we get the required result.

Theorem 3.2. Let $\mathcal{R}$ be a prime ring with $\operatorname{char}(\mathcal{R}) \neq 2, \alpha$ is an automorphism of $\mathcal{R}$ and $\beta, \gamma$ epimorphisms of $\mathcal{R}$, then the following assertions are equivalent:

(a) $\alpha(x) \circ \beta(y)+\gamma([x, y])=0$ for all $x, y \in \mathcal{R}$;

(b) $\mathcal{R}$ is commutative.

Proof. It is obvious that $(\mathrm{b}) \Rightarrow(\mathrm{a})$.

(a) $\Rightarrow$ (b) Suppose that

$$
\alpha(x) \circ \beta(y)+\gamma([x, y])=0, \quad \text { for all } x, y \in \mathcal{R} .
$$

Replacing $y$ by $y x$ in (3.20) and using identity $(c)$, we get

$$
\beta(y)[\alpha(x), \beta(x)]=(\alpha(x) \circ \beta(y)) \beta(x)+\gamma([x, y]) \gamma(x), \quad \text { for all } x, y \in \mathcal{R} .
$$

From (3.20) and (3.21) it follows that

$$
\beta(y)[\alpha(x), \beta(x)]=\gamma([x, y])(\gamma(x)-\beta(x)), \quad \text { for all } x, y \in \mathcal{R} .
$$

Putting $x y$ in place of $y$ in (3.22), we find that

$$
(\gamma(x)-\beta(x)) \gamma([x, y])(\gamma(x)-\beta(x))=0, \quad \text { for all } x, y \in \mathcal{R} .
$$


Invoking (3.22), (3.23) yields

$$
(\gamma(x)-\beta(x)) \beta(y)[\alpha(x), \beta(x)]=0, \quad \text { for all } x, y \in \mathcal{R} .
$$

Since $\beta$ is onto, we obtain

$$
(\gamma(x)-\beta(x)) \mathcal{R}[\alpha(x), \beta(x)]=\{0\}, \quad \text { for all } x \in \mathcal{R} .
$$

By primeness of $\mathcal{R}$, we get

$$
\gamma(x)=\beta(x) \quad \text { or } \quad[\alpha(x), \beta(x)]=0, \quad \text { for all } x \in \mathcal{R} .
$$

If there exists $x_{0} \in \mathcal{R}$ such that $\left[\alpha\left(x_{0}\right), \beta\left(x_{0}\right)\right]=0$, then $(3.22)$ gives $\gamma\left(\left[x_{0}, y\right]\right)\left(\gamma\left(x_{0}\right)-\right.$ $\left.\beta\left(x_{0}\right)\right)=0$ for all $y \in \mathcal{R}$. Replacing $y$ by $y r$, we get $\gamma\left(\left[x_{0}, y\right]\right) \gamma(r)\left(\gamma\left(x_{0}\right)-\beta\left(x_{0}\right)\right)=0$ for all $y, r \in \mathcal{R}$. Since $\gamma$ is onto, we obtain $\gamma\left(\left[x_{0}, y\right]\right) \mathcal{R}\left(\gamma\left(x_{0}\right)-\beta\left(x_{0}\right)\right)=\{0\}$ for all $y \in \mathcal{R}$. By primeness of $\mathcal{R}$, one can easily verify that $\gamma\left(\left[x_{0}, y\right]\right)=0$ for all $y \in \mathcal{R}$ or $\gamma\left(x_{0}\right)=\beta\left(x_{0}\right)$.

Suppose the first case and using (3.20), we get $\alpha\left(x_{0}\right) \circ \beta(y)=0$ for all $y \in \mathcal{R}$. Replacing $y$ by $y t$ and using identity $(c)$, we obtain $\beta(y)\left[\alpha\left(x_{0}\right), \beta(t)\right]=0$ for all $y, t \in \mathcal{R}$. Since $\mathcal{R}$ is prime and $\beta$ is onto, we get $\alpha\left(x_{0}\right) \in Z(\mathcal{R})$, and therefore, (3.20) forces that $2 \alpha\left(x_{0}\right) \mathcal{R} \beta(y)=0$ for all $y \in \mathcal{R}$. Using the fact that $\mathcal{R}$ is prime and $\operatorname{char}(\mathcal{R}) \neq 2$, we get $\alpha\left(x_{0}\right)=0$. Since $\alpha$ is an automorphism of $\mathcal{R}$, we obtain $x_{0}=0$. In this case, (3.24) becomes $\gamma(x)=\beta(x)$, for all $x \in \mathcal{R}$. Replacing $y$ by $x y$ in (3.20) and using it, we get

$$
\alpha(x) \circ \beta(x) \beta(y)+\beta(x)(-\alpha(x) \circ \beta(y))=0, \quad \text { for all } x, y \in \mathcal{R} .
$$

Developing the last expression, we arrive at

$$
[\alpha(x), \beta(x)] \beta(y)=0, \quad \text { for all } x, y \in \mathcal{R} .
$$

Using the fact that $\mathcal{R}$ is prime and $\beta$ is onto, we obtain $[\alpha(x), \beta(x)]=0$, for all $x, y \in$ $\mathcal{R}$. For $y=x,(3.20)$ with the last expression give $\alpha(x) \beta(x)=\beta(x) \alpha(x)=0$ for all $x \in \mathcal{R}$.

Replacing $y$ by $y x$ in (3.20) and using it again, we obtain

$$
\alpha(x) \circ \beta(y) \beta(x)+\beta([x, y]) \beta(x)=0, \quad \text { for all } x, y \in \mathcal{R} .
$$

Developing (3.25) by using identity $(c)$, we conclude that

$$
[\alpha(x), \beta(y)] \beta(x)+\beta([x, y]) \beta(x)=0, \quad \text { for all } x, y \in \mathcal{R} \text {. }
$$

Putting $y t$ in place of $y$ and using identity $(a)$ with (3.26), we can easily arrive at

$$
([\alpha(x), \beta(y)]+\beta([x, y])) \beta(t) \beta(x)=0, \quad \text { for all } x, y, t \in \mathcal{R} .
$$

Since $\beta$ is onto, equation (3.27) reduces to

$$
([\alpha(x), \beta(y)]+\beta([x, y])) \mathcal{R} \beta(x)=\{0\}, \quad \text { for all } x, y \in \mathcal{R} .
$$

By primeness of $\mathcal{R}$, we obtain

$$
[\alpha(x), \beta(y)]+\beta([x, y])=0 \quad \text { or } \quad \beta(x)=0, \quad \text { for all } x, y \in \mathcal{R} .
$$


It is clear that both cases give the following equation

$$
[\alpha(x), \beta(y)]+\beta([x, y])=0, \quad \text { for all } x, y \in \mathcal{R} .
$$

As (3.28) is the same as (3.18), arguing as in the proof of Theorem 3.1, we conclude that $\mathcal{R}$ is commutative.

In Examples 3.1, 3.2, we show that the condition " $\mathcal{R}$ is prime" is necessary in Theorems 3.1, 3.2.

Example 3.1. Let us defined $\mathcal{R}$ and $\alpha, \beta, \gamma: \mathcal{R} \rightarrow \mathcal{R}$ as follow:

$$
\begin{aligned}
& \mathcal{R}=\left\{\left(\begin{array}{lll}
x & y & 0 \\
0 & 0 & 0 \\
0 & z & 0
\end{array}\right) \mid x, y, z \in \mathbb{Z}\right\}, \quad \alpha\left(\begin{array}{lll}
x & y & 0 \\
0 & 0 & 0 \\
0 & z & 0
\end{array}\right)=\left(\begin{array}{ccc}
x & y & 0 \\
0 & 0 & 0 \\
0 & -z & 0
\end{array}\right), \\
& \beta=i d_{\mathcal{R}}, \quad \gamma\left(\begin{array}{lll}
x & y & 0 \\
0 & 0 & 0 \\
0 & z & 0
\end{array}\right)=\left(\begin{array}{ccc}
x & -y & 0 \\
0 & 0 & 0 \\
0 & z & 0
\end{array}\right) \text { and } \delta=0 .
\end{aligned}
$$

It is clear that $\mathcal{R}$ is a ring which is not prime and $\operatorname{char}(\mathcal{R}) \neq 2$. Moreover, $\alpha$ is an endomorphism of $\mathcal{R}$ and $\beta, \gamma$ epimorphisms of $\mathcal{R}$ such that $[\alpha(x), \beta(y)]+\gamma([x, y])=0$ for all $x, y \in \mathcal{R}$, but $\mathcal{R}$ is noncommutative.

Example 3.2. Let us defined $\mathcal{R}$ and $\alpha, \beta, \gamma: \mathcal{R} \rightarrow \mathcal{R}$ as follow:

$$
\begin{aligned}
& \mathcal{R}=\left\{\left(\begin{array}{lll}
x & y & 0 \\
0 & 0 & 0 \\
0 & z & 0
\end{array}\right) \mid x, y, z \in \mathbb{Z}\right\}, \quad \alpha=i d_{\mathcal{R}}, \\
& \beta=\left(\begin{array}{lll}
x & y & 0 \\
0 & 0 & 0 \\
0 & z & 0
\end{array}\right)=\left(\begin{array}{ccc}
-x & y & 0 \\
0 & 0 & 0 \\
0 & z & 0
\end{array}\right), \quad \gamma\left(\begin{array}{lll}
x & y & 0 \\
0 & 0 & 0 \\
0 & z & 0
\end{array}\right)=\left(\begin{array}{ccc}
x & -y & 0 \\
0 & 0 & 0 \\
0 & z & 0
\end{array}\right) .
\end{aligned}
$$

It is clear that $\mathcal{R}$ is a ring which is not prime and $\operatorname{char}(\mathcal{R}) \neq 2$. Moreover, $\alpha$ is an automorphism of $\mathcal{R}$ and $\beta, \gamma$ epimorphisms of $\mathcal{R}$ such that $\alpha(x) \circ \beta(y)+\gamma([x, y])=0$ for all $x, y \in \mathcal{R}$, but $\mathcal{R}$ is noncommutative.

The following example proves that the condition " $\operatorname{char}(\mathcal{R}) \neq 2$ " in Theorem 3.2 is not superfluous.

Example 3.3. Let us define $\mathcal{R}=M_{2}\left(\mathbb{Z}_{2}\right)$ and $\alpha=\beta=\gamma=i d_{\mathcal{R}}$. It is clear that $\mathcal{R}$ is a noncommutative prime ring such that $\operatorname{char}(\mathcal{R})=2$. Moreover, $\alpha$ is an automorphism of $\mathcal{R}$ and $\beta, \gamma$ epimorphisms of $\mathcal{R}$ such that

(a) $[\alpha(x), \beta(y)]+\gamma([x, y])=0$ for all $x, y \in \mathcal{R}$;

(b) $\alpha(x) \circ \beta(y)+\gamma([x, y])=0$ for all $x, y \in \mathcal{R}$.

But $\mathcal{R}$ is noncommutative. 


\section{REFERENCES}

[1] M. Ashraf, A. Ali and S. Ali, $(\sigma, \tau)$-derivations on prime near rings, Arch. Math. 40(3) (2004), 281-286.

[2] M. Ashraf and N. Rehman, On commutativity of rings with derivations, Results Math. 42(1-2) (2002), 3-8.

[3] M. Ashraf and A. Boua, On semiderivations in 3-prime near-rings, Commun. Korean Math. Soc. 31(3) (2016), 433-445.

[4] H. E. Bell and M. N. Daif, On commutativity and strong commutativity preserving maps, Canad. Math. Bull. 37 (1994), 443-447.

[5] H. E. Bell and N.-Ur Rehman, Generalized derivations with commutativity and anticommutativity conditions, Math. J. Okayama Univ. 49 (2007), 139-147.

[6] M. Bresar, On the distance of the composition of two derivations to the generalized derivations, Glasgow Math. J. 33 (1991), 89-93.

[7] A. A. Klein, A new proof of a result of Levitzki, Proc. Amer. Math. Soc. 81(1) (1981), 8.

${ }^{1}$ ABdelKarim Boua,

Sidi Mohammed Ben Abdellah University, Polydisciplinary Faculty, LSI,

TAZA, MOROCCO

Email address: abdelkarimboua@yahoo.fr 